\title{
Avaliação da confiabilidade e usabilidade de três diferentes programas computacionais para a análise fotogramétrica do ângulo de flexão de quadril Reliability and usability assessment of three different softwares for photogrammetric analysis of hip flexion angle
}

Débora Alves Guariglia ${ }^{1}$, Ligia Maxwell Pereira ${ }^{2}$, Hugo Maxwell Pereira ${ }^{3}$, Jefferson Rosa Cardoso $0^{4}$

Estudo desenvolvido no

Laboratório de Eletromiografia

Cinesiológica e Cinemática do

Departamento de Fisioterapia

da Universidade Estadual de

Londrina (UEL) - Londrina (PR),

Brasil.

${ }^{1}$ Mestre em Educação Física pela UEL-UEM.

2 Doutoranda em Educação Física pela UEL-UEM.

${ }^{3}$ Doutorando Interdisciplinar pela Marquette University - Milwaukee (WI), Estados Unidos.

${ }^{4}$ Doutor em Ciências; Professor Associado do Departamento de Fisioterapia da UEL - Londrina (PR), Brasil.

\section{ENDERECO PARA CORRESPONDÊNCIA}

Jefferson Rosa Cardoso Hospital Universitário - Av. Robert Kock, 60 - CEP 86038440 - Londrina (PR), Brasil E-mail: jeffcar@uel.br

\section{APRESENTACÃO}

out. 2010

ACEITO PARA PUBLICAÇÃO abr. 2011

FONTE DE FINANCIAMENTO nenhuma

CONFLITO DE INTERESSES nada a declarar
RESUMO: A análise fotogramétrica pode ser feita por meio de diferentes programas computacionais. Assim, o objetivo do presente estudo foi avaliar a confiabilidade e usabilidade de três diferentes programas em uma análise fotogramétrica durante a flexão do quadril e discutir aspectos qualitativos e quantitativos de cada um deles. Participaram do estudo 26 voluntários do gênero masculino, com idade entre 19 e 30 anos $(\bar{x}=25,4$ anos, $D P=2,5)$ que realizaram o teste do sentar e alcançar por meio do banco de Wells adaptado. Marcadores cutâneos foram posicionados nos processos ósseos: trocânter maior do fêmur e espinha ilíaca anterossuperior. A aquisição da imagem foi realizada no limite máximo da execução do teste. Todos os programas apresentaram uma alta confiabilidade entre as medidas, além disso, foram apresentadas vantagens e desvantagens específicas na adoção de cada um deles. O programa SAPO foi considerado com maiores vantagens para utilização na prática profissional, no entanto, para realização de análises em pesquisas científicas todos os programas apresentam pontos que devem ser considerados antes da sua adoção.

Descritores: biomecânica; amplitude de movimento articular; articulação do quadril; reprodutibilidade dos testes.

ABSTRACT: The photogrammetric analysis can be done by using different softwares. Thus, the purpose of this study was to assess the reliability and the usability of three different softwares during the hip flexion photogrammetric analysis and to discuss the qualitative and quantitative aspects of each one. Twenty-six volunteers participated in this study with age between 19 and 30 years old ( $\bar{x}=25.4$ years, $\mathrm{SD}=2.5)$ who underwent the sit-to-reach test through adapted Wells' bench. Reflective skin markers were positioned in the anterior superior iliac spine and greater trochanter. The image acquisition was collected in the maximum limit of test execution. All softwares showed a high reliability between measures, besides it was presented specifics advantages and disadvantages during adoption one of them. SAPO's software was considered with major points for professional practice, although taking the analysis for scientific research all softwares demonstrated characteristics that must be taken into consideration before adopting them.

KeYwords: biomechanics; range of motion, articular; hip joint; reproducibility of results. 


\section{INTRODUCÃO}

A análise fotogramétrica tem sido utilizada para avaliar postura e também a flexibilidade de diferentes segmentos, por isso sua padronização e validade são necessárias para obtenção de dados mais precisos e fidedignos ${ }^{1}$. Estes procedimentos têm sido objeto de investigação em inúmeros estudos que verificaram a confiabilidade ${ }^{2-7}$ e a validade ${ }^{3,8}$ desse tipo de medida. Informações na avaliação da flexibilidade foram investigadas por Bozic et al. ${ }^{3}$, os quais testaram a confiabilidade e a validade concorrente de testes usuais como o sentar e alcançar e avaliações de imagens de alta precisão no sistema de análise cinemática em 3D. Os autores verificaram que nem todos os testes de flexibilidade são concordantes com os valores observados em avaliações com imagens. lunes et al. ${ }^{4}$ também verificaram que dados da fotogrametria não podem ser correlacionados com dados de avaliação postural visual.

Para a realização da análise fotogramétrica é necessário a adoção de programas para a avaliação das imagens. Os programas mais conhecidos são o AutoCAD, SAPO e Corel Draw. O AutoCAD é um programa de desenho para a criação de projetos em duas ou três dimensões, normalmente utilizado em engenharia, arquitetura, design e áreas afins ${ }^{9}$. O programa SAPO (software para avaliação postural) foi desenvolvido especificamente para profissionais da saúde como instrumento de avaliação postural ${ }^{10}$. E por fim, o Corel Draw é um programa de desenho vetorial bidimensional desenvolvido inicialmente para designers gráficos criarem seus produtos artísticos, publicitários, logotipos, entre outros ${ }^{11}$.

Somente um dos programas comparados, o SAPO, foi construído e validado com a finalidade de realizar análises fotogramétricas ${ }^{8,10}$, porém os outros dois também são utilizados nesse modelo de investigação. No entanto, apesar dos programas seguirem pressupostos de análise semelhantes, as ferramentas para a realização da análise entre os mesmos são diferenciadas. A escolha normalmente é pautada pela familiaridade do pesquisador com o programa. Com isso, por apresentarem diferentes ferramentas e características, a adoção de um determinado programa pode proporcionar resultados diferentes, o que impediria a comparação dos dados gerados por eles.

Alguns estudos já foram realizados com a utilização dos instrumentos citados. Sacco et al. ${ }^{6}$ testaram a confiabilidade da fotogrametria computadorizada, por meio de dois programas, o Corel Draw e o SAPO, em relação à goniometria para alguns ângulos na análise postural nos membros inferiores e não encontraram diferenças entre os programas em relação aos ângulos, porém alguns valores se diferiram entre a goniometria e a fotogrametria. No entanto, esses dados referemse a análises posturais e não a análises mais especificas com flexão de quadril. Além disso, informações comparativas com o AutoCAD não foram encontradas nas investigações existentes.

Por tanto, o objetivo do estudo foi avaliar aspectos quantitativos como a confiabilidade de cada programa (SAPO, AutoCAD e Corel Draw) e qualitativos como a usabilidade, vantagens e desvantagens apresentadas por cada um deles para a realização da análise fotogramétrica de flexão do quadril.

\section{METODOLOGIA}

A amostra de conveniência foi formada por 26 voluntários do gênero masculino. Os critérios de inclusão foram indivíduos sadios com idade entre 18 e 30 anos. Foram excluídos os participantes que apresentaram alterações posturais evidentes, dores e distúrbios musculoesqueléticos. Todos os participantes foram informados dos propósitos e procedimentos do estudo e assinaram um termo de consentimento livre e esclarecido para iniciar as avaliações. O estudo foi aprovado pelo Comitê de Ética em Pesquisa da Instituição (107/08).

\section{Procedimentos}

A flexibilidade do quadril foi avaliada por análise fotogramétrica angular no teste sentar e alcançar ${ }^{12}$. Para a realização desse procedimento, foi utilizado um banco de Wells adaptado e uma câmera fotográfica digital (5 megapixels) que foi posicionada e calibrada sobre um tripé fixo de $72 \mathrm{~cm}$ de altura, a $202 \mathrm{~cm}$ do plano de fundo. Um fio de prumo foi fixado no teto e estendido até próximo ao banco para auxiliar na calibragem da foto posteriormente. A adaptação do banco consistiu em uma abertura (porta) de $27 \mathrm{~cm}$ de altura x $27 \mathrm{~cm}$ de largura no local do apoio plantar, na caixa (Figura 1) o que diminui a influência dos músculos gastrocnêmios na análise ${ }^{13}$.

A foto era registrada no momento em que o indivíduo estava em seu limite máximo na execução do teste, ou seja, era solicitado ao participante que realizasse inspiração profunda e durante a expiração flexionasse o tronco à frente em seu limite máximo com as mãos sobrepostas sobre o banco. Para evitar possíveis deslocamentos laterais, os membros superiores deveriam estar alinhados à fita métrica do banco. Além disso, não foi permitido que

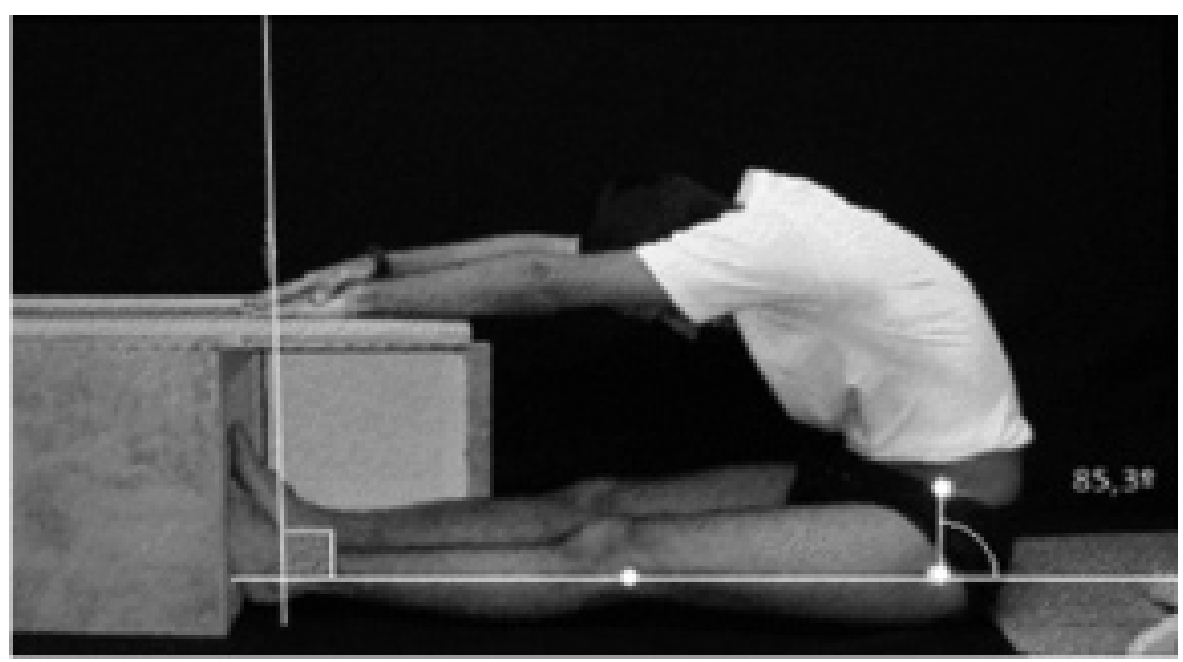

Figura 1. Banco de Wells adaptado com abertura no apoio plantar e análise fotogramétrica de quadril 
os indivíduos executassem qualquer tipo de aquecimento ou alongamento anterior aos testes. A análise fotogramétrica do quadril foi avaliada pelo ângulo oriundo de uma linha horizontal, desenhada perpendicularmente ao fio de prumo, e pelos marcadores reflexivos no corpo dos sujeitos, que foram colocados nos pontos anatômicos: trocânter maior do fêmur e espinha ilíaca anterossuperior (Figura 1).

As análises foram feitas nos programas AutoCAD, SAPO e Corel Draw por 2 avaliadores que examinaram 26 fotos duas vezes em cada programa, com pelo menos 1 dia de intervalo entre as análises dos mesmos. Um avaliador não tinha acesso ao resultado do outro avaliador. Em todos os programas, os passos para a análise das fotos são os mesmos: primeiro, calibração da foto por um ponto referencial (normalmente é adotado o fio de prumo); segundo, seleção dos marcadores e terceiro, determinação do ângulo entre os marcadores.

\section{Análise estatística}

O teste de Shapiro-Wilk foi utilizado para verificar a distribuição de normalidade dos dados. Como os pressupostos foram assumidos, os dados foram apresentados em média $(\bar{x})$ e desvio padrão (DP). Para identificar as diferenças dos resultados entre os programas, foi utilizada análise de variância para medidas repetidas a qual atendeu os pressupostos de esfericidade. Tanto a confiabilidade intra-avaliador quanto a interavaliadores foram calculadas pelo coeficiente de correlação intraclasse (efeito aleatório - um fator) e a análise de concordância proposta por Bland e Altman ${ }^{14}$. A significância estatística adotada para todas as análises foi de $5 \%(p \leq 0,05)$. Foram utilizados os seguintes programas para as análises: SPSS 15.0, MedCalc 11.3.3.

\section{RESULTADOS}

Os 26 participantes do estudo apresentaram idade média de 25,4 anos $(\mathrm{DP}=2,5)$, massa corporal de $80,3 \mathrm{~kg}$ $(\mathrm{DP}=14,6)$, estatura de 1,77 m $(\mathrm{DP}=0,06)$ e índice de massa corpórea (IMC) de $25,3 \mathrm{~kg} / \mathrm{m}^{2}(\mathrm{DP}=4,0)$. Na Tabela 1, encontram-se os valores da análise fotogramétrica do ângulo do quadril dos três programas.

Tabela 1. Média (desvio padrão) das análises do ângulo do quadril em cada programa

\begin{tabular}{lll}
\hline & Avaliador 1 & Avaliador 2 \\
\hline AutoCAD & $80,3^{\circ}(19,5)$ & $80,3^{\circ}(19,6)$ \\
SAPO & $80,5^{\circ}(19,7)$ & $80,5^{\circ}(19,5)$ \\
Corel Draw & $80,1^{\circ}(19,4)$ & $79,8^{\circ}(19,0)$ \\
\hline
\end{tabular}

Na Tabela 2 são apresentados os valores do teste de confiabilidade intra e interavaliador da análise da articulação do quadril nos três diferentes programas. As análises apresentaram alta confiabilidade $(\mathrm{CCl}$ entre 0,99 e 1,00) e (Bland e Altman ${ }^{14}$ entre $-0,23$ e 0,38$)$.

A Figura 2 apresenta uma comparação dos resultados obtidos entre os programas, a qual não demonstrou diferenças estatisticamente significantes entre os programas e entre os avaliadores $(F=1,381 ; p=0,257)$. Na Tabela 3, são apresentadas vantagens e desvantagens dos três programas.

\section{DISCUSSÃO}

O presente estudo foi elaborado para avaliar uma melhor opção na utilização de diferentes programas computacionais por meio de informações qualitativas e dados quantitativos gerados pelos mesmos. Com isso foi possível determinar o mais confiável para a realização das análises e verificar se é aceitável comparações das investigações com valores gerados.

Tabela 2. Teste de confiabilidade das análises fotogramétricas entre os avaliadores nos programas adotados

\begin{tabular}{|c|c|c|c|c|}
\hline \multicolumn{2}{|c|}{$\mathrm{CCl}$ (efeito aleatório - um fator) } & \multicolumn{3}{|c|}{ Bland e Altman ${ }^{14}$} \\
\hline & $\mathrm{CCl}-\mathrm{IC} 95 \%$ & $\bar{d}$ & DP da $\bar{d}$ & IC95\% da $\bar{d}$ \\
\hline \multicolumn{5}{|l|}{ AutoCAD } \\
\hline Intra-avaliador 1 & $1,0(1,00-1,00)$ & 0,03 & 0,52 & $-0,17 ; 0,25$ \\
\hline Intra-avaliador 2 & $1,0(0,99-1,00)$ & 0,11 & 0,65 & $-0,14 ; 0,37$ \\
\hline Interavaliadores & $1,0(1,00-1,00)$ & $-0,03$ & 0,77 & $-0,35 ; 0,27$ \\
\hline \multicolumn{5}{|l|}{ SAPO } \\
\hline Intra-avaliador 1 & $0,99(0,99-0,99)$ & 0,38 & 0,98 & $-0,01 ; 0,78$ \\
\hline Intra-avaliador 2 & $0,99(0,99-0,99)$ & 0,38 & 1,62 & $-0,27 ; 1,04$ \\
\hline Interavaliadores & 0,99 (0,99-0,99) & 0,000 & 1,32 & $-0,53 ; 0,53$ \\
\hline \multicolumn{5}{|l|}{ Corel Draw } \\
\hline Intra-avaliador 1 & $0,99(098-0,99)$ & $-0,23$ & 2,00 & $-1,07 ; 0,61$ \\
\hline Intra-avaliador 2 & $0,99(0,99-0,99)$ & 0,07 & 1,97 & $-0,72 ; 0,87$ \\
\hline interavaliadores & $0,99(0,98-0,99)$ & 0,03 & $-0,30$ & $-1,26 ; 0,65$ \\
\hline
\end{tabular}

$\overline{\mathrm{d}}$ : diferença da média; DP: desvio padrão; IC: intervalo de confiança; $\mathrm{CCl}$ : coeficiente de correlação intraclasse
Para verificação da confiabilidade foi adotada tanto o coeficiente de correlação intraclasse $(\mathrm{CCl})$ quanto o teste de concordância de Bland e Altman ${ }^{14}$ visto que este procedimento estatístico associado é recomendado, pois o $\mathrm{CCl}$ isoladamente não promove informação suficiente sobre a confiabilidade das medidas devido à influência da magnitude da variação entre os sujeitos do valor medido ou suas variações e aos erros na medida, o que torna impossível de ser interpretado clinicamente ${ }^{15}$.

O teste de Bland e Altman ${ }^{14}$ fornece os valores do intervalo de confiança para a diferença da média e os limites de concordância que indicarão os erros das medidas e poderão relacionar a aceitabilidade prática das mesmas ${ }^{13}$. Para interpretação dessa análise, é considerada a melhor concordância a medida cuja zero. Ao considerar o grau de concordância, todos os programas apresentaram valores excelentes $(\bar{d}<0,4)$.

Em relação aos valores do $\mathrm{CCl}$, verificou-se que todos os programas apresentaram valores iguais ou superiores a diferença da média $(\bar{d})$ se aproxima de 
0,99 tanto intra-avaliadores como interavaliadores. No entanto, o AutoCAD foi o único que apresentou em todas as análises valores de $\mathrm{CCl}=1,0$. Sacco et al. ${ }^{4}$

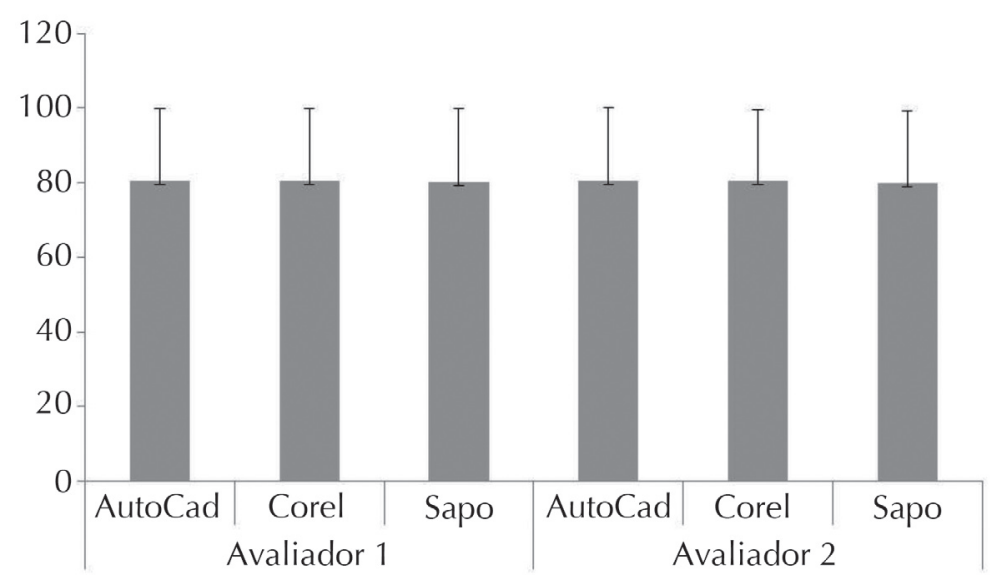

Figura 2. Média e desvio padrão (em graus) das análises entre os avaliadores testaram a confiabilidade dos programas Corel Draw e SAPO durante a análise fotogramétrica de avaliação postural e encontraram correlações fortes entre as

Tabela 3. Aspectos qualitativos em relação aos programas adotados

\begin{tabular}{|c|c|c|}
\hline Programa & Vantagens & Desvantagens \\
\hline \multirow[t]{4}{*}{ AutoCAD } & $\begin{array}{l}\text { Em um mesmo documento, consegue analisar } \\
\text { inúmeras fotos }\end{array}$ & Não foi desenvolvido para área de saúde \\
\hline & Facilidade para ampliar a foto e localizar marcadores & $\begin{array}{l}\text { Usualmente profissionais de saúde necessitam de } \\
\text { familiarização com as ferramentas }\end{array}$ \\
\hline & Conhecido internacionalmente & $\begin{array}{l}\text { Executável somente no sistema operacional } \\
\text { Microsoft Windows }\end{array}$ \\
\hline & & Indisponível gratuitamente \\
\hline \multirow[t]{7}{*}{ SAPO } & $\begin{array}{l}\text { Download gratuito do programa disponível na } \\
\text { internet }\end{array}$ & Analisa somente uma foto por vez \\
\hline & & Pouco conhecido internacionalmente \\
\hline & $\begin{array}{l}\text { Layout do programa construído para profissionais de } \\
\text { saúde }\end{array}$ & \\
\hline & $\begin{array}{c}\text { Possui possibilidades de avaliação postural pré- } \\
\text { configuradas }\end{array}$ & \\
\hline & Possui validação ${ }^{8}$ & \\
\hline & $\begin{array}{l}\text { Pode ser instalado em qualquer sistema operacional } \\
\text { (Microsoft Windows, Unix, Linux, Mac OS). }\end{array}$ & \\
\hline & $\begin{array}{c}\text { Requer menor espaço em disco rígido para } \\
\text { instalação }\end{array}$ & \\
\hline \multirow[t]{3}{*}{ Corel Draw } & $\begin{array}{l}\text { Em um mesmo documento, consegue analisar } \\
\text { inúmeras fotos }\end{array}$ & $\begin{array}{l}\text { Executável somente no sistema operacional } \\
\text { Microsoft Windows }\end{array}$ \\
\hline & Conhecido internacionalmente & $\begin{array}{l}\text { Usualmente profissionais de saúde necessitam de } \\
\text { familiarização com as ferramentas }\end{array}$ \\
\hline & & Indisponível gratuitamente \\
\hline
\end{tabular}


Medidas de amplitude articular feitas por meio de análise fotogramétrica com programas que demonstraram alta confiabilidade melhoram a cientificidade das avaliações musculoesqueléticas. O presente estudo demonstrou três diferentes programas com alta confiabilidade das medidas e de fácil aplicação. O tempo das análises para cada um deles é inferior a dois minutos por foto. No entanto, deve-se considerar que ambos os avaliadores já tinham experiência prévia com esses programas o que pode ter proporcionado uma maior velocidade de análise e melhor utilização das ferramentas.

Os dados quantitativos garantiram a confiabilidade dos três programas e a possibilidade de comparações entre eles, no entanto em uma análise qualitativa (Tabela 3 ) o AutoCAD e o Corel Draw não apresentaram as maiores vantagens, visto que não são disponíveis gratuitamente, não são programas criados para análise postural, ou seja, é necessário que o avaliador conheça previamente suas ferramentas para adaptá-las em uma análise necessária e não são executáveis em qualquer sistema operacional. Em relação aos aspectos qualitativos, o programa mais acessível é o SAPO, visto que suas ferramentas são de fácil manuseio, não há custo na aquisição e sua confiabilidade e acurácia foram testadas e aceitas ${ }^{8}$.

Além disso, todos os programas oferecem ótimas opções para medida angular, no entanto, o SAPO oferece a possibilidade de um vasto relatório postural e o programa foi desenhado para profissionais de saúde. Qualitativamente isso pode ser considerado como uma vantagem em relação aos outros programas.

\section{CONCLUSÕES}

Todos os programas mostraram-se confiáveis e comparáveis entre si, no entanto, para a adoção de um deles, é necessário levar em consideração o objetivo e sua utilização. Para a prática profissional, o SAPO apresenta maiores vantagens sobre os outros programas, visto que tem um formato voltado para essas análises, apresenta outras opções de análises, fácil utilização e está disponível gratuitamente. No entanto, para a utilização desses programas para pesquisas, é necessária uma análise pelo pesquisador sobre as vantagens de cada um deles para que a melhor escolha seja realizada.

\section{REFERÊNCIAS}

1. Fedorak C, Ashworth N, Marshall J, Paull H. Reliability of the visual assessment of cervical and lumbar lordosis: How good are we? Spine. 2003;28(16):1857-9.

2. Cardoso JR, Azevedo NCT; Cassano CS; Kawano MM; Âmbar G. Confiabilidade intra e interobservador da análise cinemática angular do quadril durante o teste sentar e alcançar para mensurar o comprimento dos isquiotibiais em estudantes universitários. Rev Bras Fisioter. 2007;11(2):133-8.

3. Bozic PR, Pazin NR, Berjan BB, Planic NM, Cuk ID. Evaluation of the field tests of flexibility of the lower extremity: reliability and the concurrent and factorial validity. J Strength Cond Res. 2010;24(9):2523-31.

4. Iunes DH, Bevilaqua-Grossi D, Oliveira AS, Castro FA, Salgado HS. Análise comparativa entre avaliação postural visual e por fotogrametria computadorizada. Rev Bras Fisioter. 2009;13(4):309-15.

5. Santos MM, Silva MPC, Sanada LS, Alves CRJ. Análise postural fotogramétrica de crianças saudáveis de 7 a 10 anos: confiabilidade interexaminadores. Rev Bras Fisioter. 2009;13(4):350-5.

6. Sacco, ICN, Alibert S, Queiroz BWC, Pripas D, Kieling I, Kimura AA, et al. Confiabilidade da fotogrametria em relação a goniometria para avaliação postural de membros inferiores. Rev Bras Fisioter. 2007;11(5):411-17.

7. Carregaro RL, Silva LCCB, Gil Coury HJC. Comparação entre dois testes clínicos para avaliar a flexibilidade dos músculos posteriores da coxa. Rev Bras Fisioter. 2007;11(2):139-45.
8. Ferreira EA, Duarte M, Maldonado EP, Burke TN, Marques AP. Postural assessment software (PAS/ SAPO): Validation and reliability. Clinics (São Paulo). 2010;65(7):675-81.

9. AutoCAD, Informações do produto. [citado 2011 Fev 06]. Disponível em: http://images.autodesk.com/latin_am_ main/files/autocad_2011_brochure.pdf

10. Ferreira EAG. Postura e controle postural: desenvolvimento e aplicação de método quantitativo de avaliação postural. [tese]. São Paulo: Faculdade de Medicina da Universidade de São Paulo, 2006.

11. CorelDRAW Graphics Suite X5, Visão geral do produto. [citado 2011 Fev 06].Disponível em: http://www.corel.com/content/pdf/cdgsx5/ CDGSX5_reviewers_guide_bp.pdf

12. Wells KF, Dillon EK. The sit and reach: a test of back and leg flexibility. Res Q Exerc Sport. 1952;23:115-8.

13. Kawano MM, Ambar G, Oliveira BIR, Boer MC, Cardoso APRG, Cardoso JR. Influence of the gastrocnemius muscle on the sit-and-reach test assessed by angular kinematic analysis. Rev Bras Fisioter. 2010;14(1):1:10-5.

14. Bland JM, Altman DG. Statistical methods for assessing agreement between two methods of clinical measurements. Lancet. 1986;1(8476):307-10.

15. Rankin G, Stokes M. Reliability of assessment tools in rehabilitation: an illustration of appropriate statistical analyses. Clin Rehabil. 1998;12(3):187-99.

16. Vincent WJ. Statistics in Kinesiology. Champaign: Human Kinetics Books, 2005. 